\title{
Science of Synthesis: Photocatalysis in Organic Synthesis
}

Edited by Burkhard König, 1st ed., Thieme: Stuttgart, 2019, 736 pp, € 259.99, ISBN: 9783132417038

Visible-light photoredox catalysis is a very powerful strategy for the generation of high-energy and reactive radical intermediates under mild conditions, thereby unlocking reaction pathways that are unattainable with traditional routes. The field has attracted huge attention in the last decade as a prominent technology in organic synthesis.

This excellent volume of Science of Synthesis: Photocatalysis in Organic Synthesis, edited by Burkhard König, represents one of the most comprehensive overviews of photocatalysis, merging fundamental principles with contemporary examples from internationally leading experts in the field.

The individual contributions are complemented by highly useful experimental sections, illustrating typical procedures for numerous photocatalytic reactions.

The book opens with a general overview of the conceptual background required to understand the field of photocatalysis, followed by a chapter focusing on practical aspects for conducting photochemical processes. These two chapters will be very useful, not only for newcomers in the field, but also for experienced researchers. The next three chapters cover photocatalytic oxidative $\mathrm{C}-\mathrm{C}$ bond formation, decarboxylative coupling reactions, and proton-coupled electron transfer processes. Attention is then turned to recent advances in enantioselective amine-catalyzed reactions trigged by light, where the synthetic opportunities opened up by the application of organocatalysis within photochemical and radical pathways are discussed.

Chapter 8 covers the reactivity of copper(I) and copper(II) complexes as powerful alternatives to common photocatalysts. Three chapters dedicated to metallaphotocatalysis, the merger of transition-metal catalysis and photocatalysis, then follow. In this context, the combination of gold, palladium, and nickel with photocatalysis are carefully presented and discussed.
The main focus of Chapters 12-14 is photocatalysis with organic dyes, also known as organophotocatalysis, where the most important photocatalytic transformations mediated by acridinium dyes and quinones (Chapter 12) and flavins (Chapter 13) are reviewed. The use of organic dyes in photocatalytic reductive $\mathrm{C}-\mathrm{H}$ arylations is discussed in Chapter 14.

While Chapter 15 describes the use of silicates as alkyl radical sources under photoredox conditions, Chapters 16-18 highlight the synthetic importance of photocatalysis in cycloadditions, carbon-heteroatom bond formation, and the introduction of fluorinated groups.

Heterogeneous photocatalysis is discussed in Chapter 19. In this context, the use of materials based on $\mathrm{TiO}_{2}$, inorganic metal oxide semiconductors, and inorganic chalcogenides for applications in organic synthesis are reported.

The last chapter was written by authors from industry (Merck) and demonstrates the use of photocatalysis in drug development.

In short, given the wealth of information and the detailed experimental procedures, this book can serve as an excellent reference source for researchers working in the area of photocatalysis as well as for organic and organometallic chemists in academia and industry. It is heartily recommended!

\section{Sami Lakhdar}

CNRS/Université Toulouse III - Paul Sabatier, Laboratoire Hétérochimie Fondamentale et Appliquée, LHFA UMR 5069, 31062 Toulouse, France

lakhdar@chimie.ups-tlse.fr 